\title{
Protocol over Things: A Decentralized Programming Model for the Internet of Things
}

\author{
Samuel H. Christie V, Daria Smirnova, Amit K. Chopra, Munindar P. Singh
}

\begin{abstract}
Current programming models for developing Internet of Things (IoT) applications conceive of an application as an orchestration. An orchestration even if physically distributed is logically centralized and thus ill-suited to the most interesting IoT applications, which involve multiple autonomous parties.

We contribute Protocols over Things (PoT), a decentralized programming model for IoT applications that represents an IoT application via a protocol between the parties involved. Notably, PoT works over unordered delivery mechanisms such as UDP and supports application-level reliability mechanisms such as resending messages.

We realize PoT using Node-RED, a popular IoT framework, to show how PoT simplifies implementation and avoids errors. Further, we empirically demonstrate that by supporting application-level retry policies, PoT provides improved performance over network-level delivery guarantees.
\end{abstract}

Index Terms-Protocol; Autonomy; Asynchrony; Endto-End Argument; Agents

\section{INTRODUCTION}

The Internet of Things (IoT) enables new applications that leverage the capabilities of things-Internetaccessible devices that sense or control their environment. Many such applications involve autonomous parties who share information from and control over things to effectively cooperate in achieving their respective goals. For example, a patient could share information with a healthcare provider to receive automatically dispensed medication. Patients should have control over their health information, and doctors over how they prescribe medications. More mundane IoT applications exhibit multiple parties too. For example, a smart home could involve an owner, one or more tenants, a power utility, and a security service. Likewise, in an enterprise, different suborganizations have different responsibilities and powers. That is, real-life applications involve multiple logical loci of control.

However, current programming models are geared toward a single locus of control: they violate the autonomy of all but one of the parties and don't jibe with things often being small and numerous. For instance, popular frameworks such as Node-RED (https://nodered.org/) and Eclipse Kura (https://www.eclipse.org/kura/) model an IoT application as an orchestration [5] that receives information from sensors, processes it in a workflow, and effects actions in the environment.

In contrast, we advocate a programming model called Protocols over Things (PoT) that is not only distributed physically but also decentralized [11]: It reflects everyone's autonomy and decouples their reasoning through asynchronous communication. Specifically, PoT captures a decentralized application via a protocol that specifies the communication constraints between the parties, abstracted as roles. PoT can employ IoT communication standards such as MQTT [4] and CoAP [8] as well as UDP [6].

Using a logistics scenario that we implement in NodeRED according to PoT, we show how a protocol specification enables the use of code generation tools and a generic communication adapter to simplify development. We focus specifically on how the adapter correlates messages according to the protocol, and compare PoT to existing approaches for message correlation in NodeRED.

We further demonstrate how PoT embodies the endto-end principle [7] by supporting deployment over unreliable asynchronous communication mechanisms instead of relying on transport-layer delivery guarantees.

We experimentally demonstrate that a PoT implementation over UDP with application-level retry strategies compares favorably with the same implementation over MQTT.

\section{EXAMPLE IOT SCENARIO: LOGISTICS}

We adopt a warehouse logistics scenario [9], simplified to focus on the aspects relevant to decentralization: parties, their communications, and their decision making.

Figure 1 illustrates this scenario conceptually. Here, Merchant, Wrapper, Labeler, and Packer are autonomous parties; the arrows indicate information flows. MERCHANT receives a purchase order (PO) imagine an external customer. Each PO includes one or more items and a shipping address. MERCHANT sends the address to LABELER, who generates the appropriate shipment label to be affixed to the shipping 
box. LABELER sends the generated label to PACKER. MERCHANT sends information about the items to the WRAPPER, who wraps the items appropriately for shipping (e.g., paper for durable items and bubble wrap for fragile ones) and notifies PACKER they are ready. PACKER affixes the shipment label to a box and notifies Merchant for each item (in the PO) that it packs in the box.

Each party applies its decision making in deciding when and what information to communicate. For example, WRAPPER may select a wrapping based on current inventory and cost and LABELER may select a shipping label based on the speed and cost of the shipper. Further, a party may choose to delay or not to send a message; e.g., WRAPPER may hold on to an item until it has the appropriate wrapping, and LABELER may discard invalid addresses. In general, the parties work concurrently and asynchronously based on information available to them.

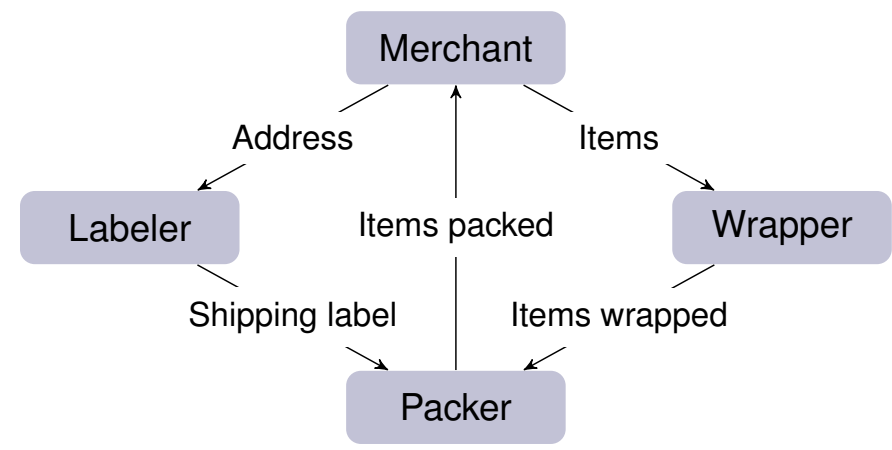

Fig. 1. Conceptual model of the logistics scenario, relative to a single purchase order (PO).

\section{SIDEBAR: NODE-RED}

Node-RED is an interactive programming and execution environment for IoT applications. A Web interface presents a palette of function blocks called nodes. A user can construct a flow-technically an orchestrationby connecting nodes via virtual wires that run from an output port of one node to the input port of another. A flow typically starts with one or more nodes that sense information from the environment and ends with some action in the environment.

Figure 2 illustrates a flow. The type of each node is indicated by its color and icon. Each node has an informal name.

In Figure 2, the nodes named labeled and wrapped are MQTT subscriptions to the topics Labeled and Wrapped, respectively. The output ports of both labeled and wrapped are connected to the input port of the Join node, meaning that received messages are passed on to the Join node. A join node

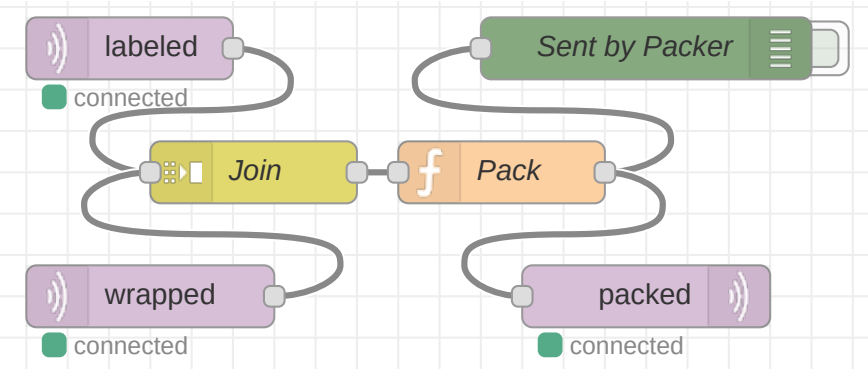

Fig. 2. A simple Node-RED flow using Join to combine messages

aggregates multiple messages together; in this example, one message from each of the MQTT topics. Pack is a function node that applies custom JavaScript code to the aggregates produced by Join. The output port of Pack is connected to two other nodes, which means that each of its outputs is copied and passed to those nodes separately. The node named Sent by Packer $\operatorname{logs}$ its inputs to the developer console, to facilitate debugging. The node named packed is an MQTT node that publishes any input it receives to the Packed topic.

\section{Challenges in Programming Iot APPLICATIONS AS ORCHESTRATIONS}

Node-RED (see Sidebar) epitomizes the orchestration approach to programming IoT applications. How might one implement a decentralized IoT application using flows? Each party's computations may be captured in a separate flow called the party's endpoint that communicates with the endpoints of other parties via messages over e.g. MQTT.

Figure 3 illustrates the resulting architecture schematically. Notably, there is no representation of the application that captures its decentralized nature except as endpoints implemented in Node-RED.

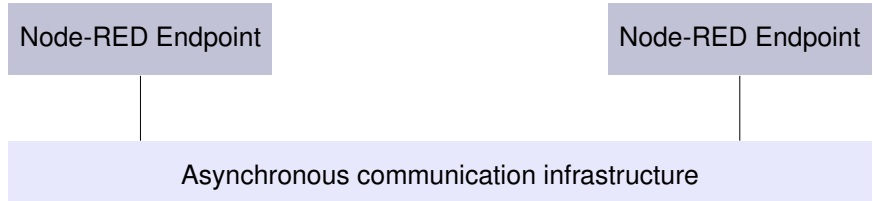

Fig. 3. Endpoint-oriented architecture for IoT applications.

There is another shortcoming in the architecture in Figure 3. To interoperate, the parties need a specification of the structures of the messages they communicate. Such a specification lies strictly outside Node-RED but is crucial to decentralization. Lacking such a specification, the endpoints would become tightly coupled. 
Listing 1. Structures of messages exchanged between the endpoints in the logistics scenario.

//Sender to Receiver: MessageName(parameter $1, \ldots$, parameter $n$ )

Merchant to Labeler: RequestLabel(orderID, address)

Merchant to Wrapper: RequestWrapping(orderID, itemID, item)

Wrapper to Packer: Wrapped(orderID, itemID, item, wrapping)

Labeler to Packer: Labeled(orderID, address, label)

Packer to Merchant: Packed(orderID, itemID, wrapping, label, status)

Suppose such a specification is available, as is common in practice. For example, Listing 1 specifies the structures of the messages alluded to in Figure 1. Sadly, the message structures in Listing 1 are inadequate for capturing the scenario; particularly its correlation requirements. PACKER needs application-level knowledge to correlate the items and shipping label for the same PO and pack them correctly, since it receives them separately.

Since Node-RED as a general-purpose platform provides only low-level programming facilities unaware of the application semantics, an implementation of correlation using standard Node-RED features will at best be ad hoc and difficult to maintain. Below, we describe three increasingly sophisticated approaches for implementing the correlation necessary for PACKER using standard Node-RED facilities, to explain their advantages and limitations. Working Node-RED flows implementing the logistics scenario with each of the approaches are available along with the rest of our code at https://gitlab.com/ masr.

\section{A. Join-Based}

Figure 2 in fact shows an endpoint for PACKER. Recall that Join in the figure produces an aggregate consisting of one message from each of the labeled and wrapped MQTT subscriptions.

Unfortunately, Join aggregates the messages solely based on their sequence in each topic-and ignores their content-producing incorrect aggregations if the label and item do not actually correlate.

For example, if PACKER receives Labeled with orderID $\mathrm{O}_{1}$ and Wrapped with orderlD $\mathrm{O}_{2}$, Join would aggregate them and misdirect the wrapped item to the address from $\mathrm{O}_{1}$ instead of $\mathrm{O}_{2}$.

Further, Join "consumes" each incoming message so it can appear in at most one output. Hence, only a single item can be associated correctly with a label. If any PO contains multiple items, Join matches the remaining items with labels from subsequent POs, causing all subsequent items to correlate with the wrong label.

\section{B. Wait-Paths-Based}

Unlike Join, wait-paths (a community plugin node) supports a correlation field, here orderID. An explicit correlation field provides some support for correct matching.

However, wait-paths also "consumes" what it correlates and associates at most one item in a PO with the PO's label. Thus, excess items are silently held (and eventually dropped) waiting for another matching label.

\section{Custom}

The Node-RED function node enables custom implementations. There are no limitations on the correctness or efficiency of a custom implementation, but such implementations would be low-level and complex.

\section{Shortcomings Identified}

First, although integrity constraints on correlation are explicit in the informal description of the scenario in Section [I] Listing 1 omits them. Representing constraints would enable reasoning about them computationally.

Second, traditional implementations entangle decision making internal to an endpoint (e.g., PACKER deciding whether, when, and what item to pack) with public communication constraints, e.g., to correlate items and labels.

Third, the endpoints may become inadvertently coupled by accounting for each other's implementation idiosyncrasies, e.g., by accommodating one box per order but failing when the order is split.

\section{The Pot Programming Model}

The foregoing shortcomings motivate a new programming model. Whereas traditional approaches focus on endpoints (as Section IV shows), PoT begins with a protocol: a specification that captures the interactions in an application via constraints on communications between the application's endpoints. 


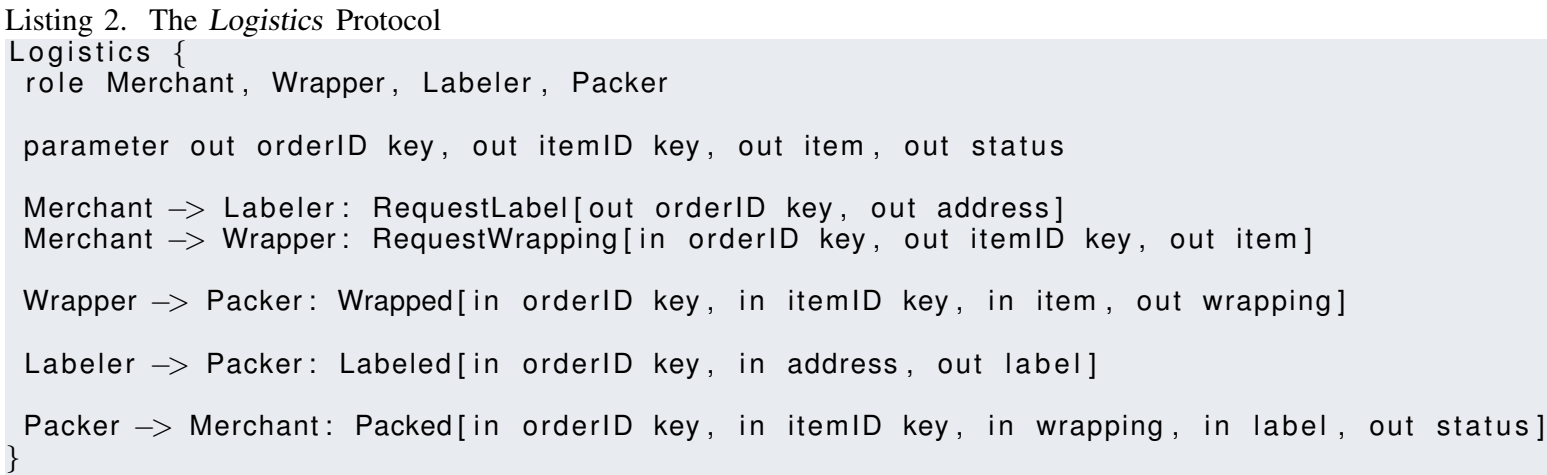

Figure 4 shows our protocol-based IoT application architecture. Here, an agent is an endpoint that adopts a role in a protocol and sends and receives messages to and from other agents in accordance with the protocol.

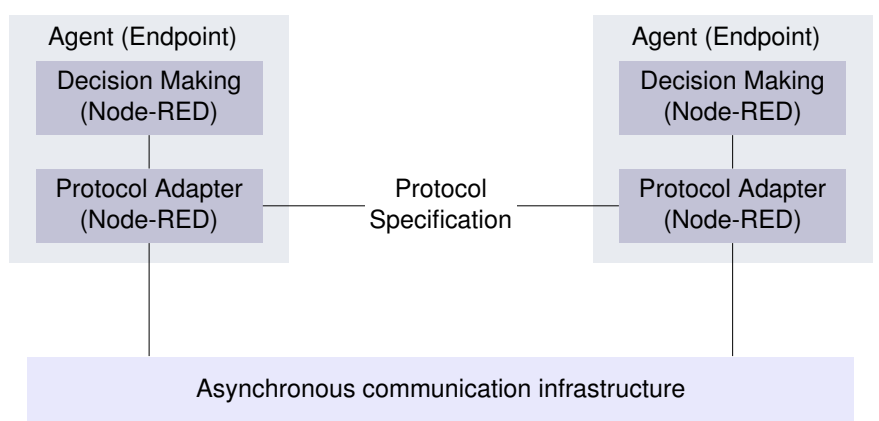

Fig. 4. The PoT architecture for IoT applications.

The Decision Making component implements an agent's private reasoning, and relies upon the Protocol Adapter to handle incoming and outgoing messages. The Adapter verifies whether messages respect the relevant causality and integrity constraints, and discards any noncompliant messages - an outgoing message may not be emitted and an incoming message may not be made available to Decision Making. Notably, the Adapter is generic and need only be supplied with the protocol specification.

\section{A. Specifying Protocols}

PoT adopts BSPL, the Blindingly Simple Protocol Language [10]. Listing 2 specifies our logistics scenario in BSPL. Here, a protocol is a bag of message schemas. A protocol involves two or more roles; Logistics involves Merchant, LABeler, Wrapper, and PACKer. A protocol has parameters; the idea is that when agents enact a protocol (by sending and receiving messages), they compute tuples of bindings for its parameters. Logistics' parameters are orderID, itemID, item, and status; enacting Logistics computes 〈orderID, itemID, item, status〉 tuples.

One or more protocol parameters are key parameters and jointly specify the key of the protocol; parameters orderID and itemID constitute the key in Logistics. A protocol's key specifies the integrity of the tuples computed by the protocol: at most, one tuple exists for a key binding. Integrity implies that a parameter may be bound only once relative to its key. Each distinct binding for a protocol's key corresponds to a distinct enactment of the protocol. A full tuple of bindings for the protocol's parameters corresponds to a complete enactment.

To capture causality constraints, a protocol's parameter is adorned $\ulcorner$ in $\urcorner$ if the protocol depends upon another protocol (via composition) for the parameter's binding; a protocol's parameter is adorned $\ulcorner$ out $\urcorner$ if the protocol itself generates its binding. Logistics' parameters are all adrorned $\ulcorner$ out $\urcorner$, meaning that enacting Logistics generates bindings for them all.

A message schema is an elementary protocol. Every message schema has a sender and a receiver role. Each message schema has parameters that are adorned $\ulcorner$ in $\urcorner$ or $\ulcorner$ out $\urcorner$, and some of which constitute a key. The sender may send a message (instance) of the (message) schema if it knows (from prior interactions) the bindings of all parameters adorned $\ulcorner$ in $\urcorner$ in the schema and does not know the bindings of any parameter adorned $\ulcorner$ out $\urcorner$ in the schema. In sending the message, the sender can generate any binding for an $\ulcorner$ out $\urcorner$ parameter. Thus, e.g., WRAPPER must know the bindings of orderID, itemID, and item before sending a Wrapped message, but may generate a binding of wrapping.

In keeping with asynchrony, there are no constraints on when a message may be received; no ordering guarantees are required from the communication infrastructure.

Parameter bindings become known to agents only through message emissions and receptions; there is no shared storage.

Formalizing protocols yields three benefits. First, pro- 


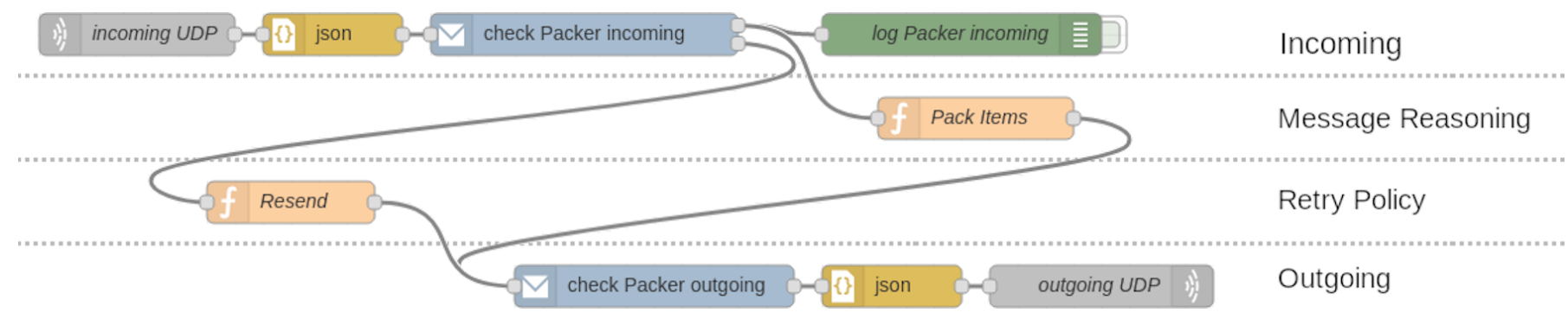

Fig. 5. PoT version of Packer flow, showing the architectural layers.

tocols separate decision making from interaction. Second, with BSPL specifically, integrity constraints capture correlation requirements declaratively. For example, the bindings of wrapping and label in a Packed message must be consistent with the binding of 〈orderID, itemID (Packed's key). Finally, as we discuss further in the next section, a clear specification of the communication constraints supports the implementation of agents by enabling the automatic generation of agent skeletons that enforce those constraints with the help of a generic protocol adapter.

\section{IMPLEMENTING PoT AGENTS}

Figure 5 shows the implementation of a PACKER agent according to the PoT model. The flow is separated into four layers: Incoming, Message Reasoning, Retry Policy, and Outgoing. The Incoming and Outgoing layers jointly constitute the protocol adapter component of the architecture, but are separated because of the one-way flow programming style of Node-RED. Message Reasoning and Retry Policy together constitute the decision-making component; they handle new messages and duplicate messages, respectively.

Given a protocol, our tooling generates a skeleton consisting of the Incoming and Outgoing layers for every role in it. For every agent playing a role, the Message Reasoning and Retry Policy parts are added by the agent developer. We describe each layer below.

1) Incoming: This layer implements the reception half of an agent's protocol adapter.

Nodes incoming UDP and json receive messages over UDP and decode them from JSON, respectively. Node check Packer incoming has access to the agent's history, which is a collection of messages that the agent has already observed. If an incoming message is not a duplicate and satisfies integrity, check Packer incoming adds it to the history and outputs it via the top output port. If the message is a duplicate, the node outputs it via the bottom output port without adding it to the history again. Integrity checking on reception is a defense against agents who may send messages in violation of integrity.

Node log Packer incoming optionally logs received messages to the developer console.

2) Message Reasoning: This layer captures an agent's reasoning, as the developer deems fit, as it reacts to received messages and potentially generates outgoing messages. In Figure 5, the PACKER implementation uses a single function node Pack Items to send Packed messages in reaction to received RequestLabel and RequestWrapping messages. Outgoing messages may also be produced in response to other events; for example, MERCHANT could initiate Logistics based on POs in a database.

3) Retry Policy: PoT also enables support for agentspecific retry policies for handling failure cases such as lost messages, and reacting to duplicate incoming messages. If an agent expects to receive a future message in response to one that it sends, such as MERCHANT expecting a Packed message for each item, it can detect possible message loss when those expectations are not met within a specified time. Our MERCHANT implementation repeatedly resends the RequestLabel and RequestWrapping messages for an item every second until the corresponding Packed message is received.

However, resending a message only addresses the loss of that message; the loss of Wrapped, Labeled or Packed would also prevent MERCHANT from receiving Packed. To further support recovery, the other agents can resend relevant messages when they receive a duplicate. Our Node-RED implementation supports this pattern by providing a second output port on the reception checking node for handling duplicate messages. In our implementation of PACKER, the Resend node handles duplicate Labeled or Wrapped messages and resends the corresponding Packed message if available.

PoT's support for agent retry policies reflects the endto-end principle [7], providing application-specific assurance of correctness, without the overhead of redundant protections in lower layers of the infrastructure. 


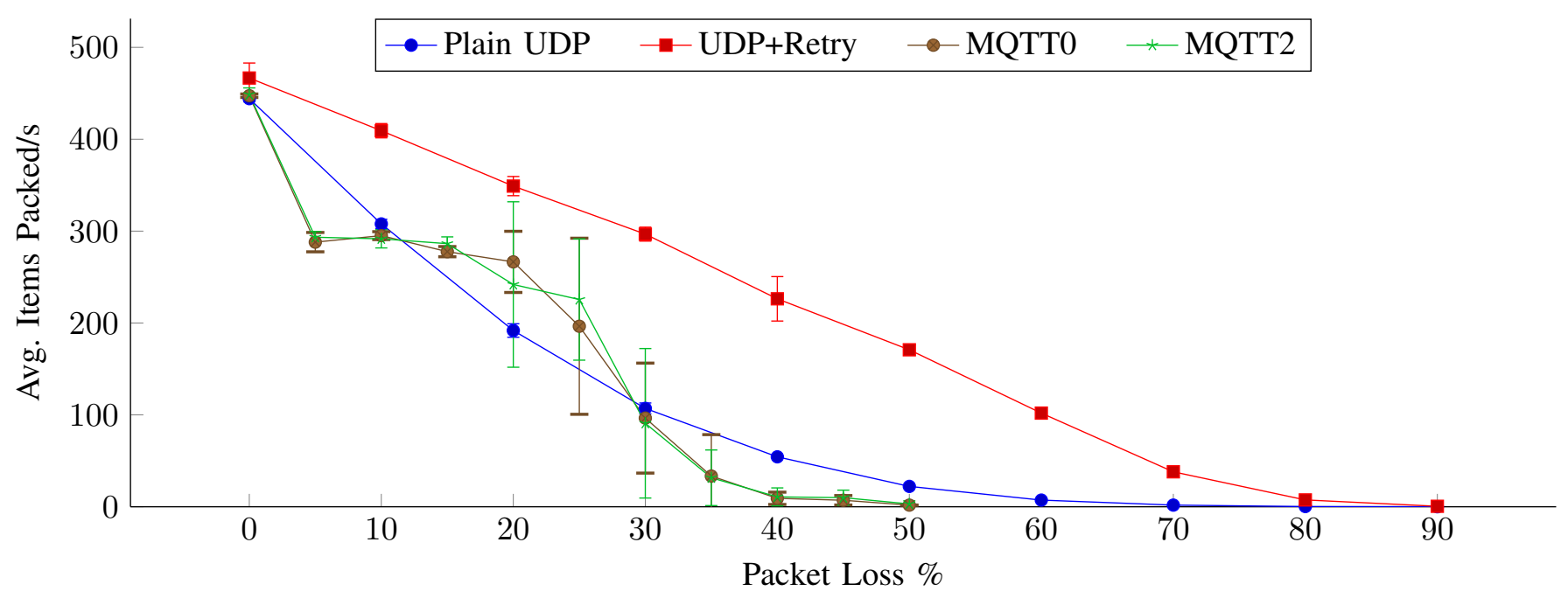

Fig. 6. A comparison of different retry policies under different packet loss rates with respect to transaction completion.

4) Outgoing: This layer implements the emission half of an agent's protocol adapter. The layer checks every outgoing message, adding to the agent's history and sending over the network those that satisfy the relevant causality and integrity constraints, and dropping those that do not. In Figure 5, the check Packer outgoing node performs the checking. To support retry policies, the node allows sending duplicates; however, they are not added to the agent's history.

Node json encodes the message in JSON and outgoing UDP sends it via UDP to the specified recipient.

\section{EVALUATion}

To demonstrate the advantages of PoT and its support for application-level retry policies, we evaluated four variations of our PoT-based implementation of the Logistics scenario.

Plain UDP. PoT over UDP, without retries.

UDP+Retry. PoT over UDP, with the above-mentioned retry policies.

MQTTO. PoT over MQTT at QoS level 0.

MQTT2. PoT over MQTT at QoS level 2.

MQTT runs over TCP. QoS level 0 and level 2 mean that MQTT guarantees a message will be delivered at most once and exactly once, respectively.

We set up our experiment to compare the variations by their average throughput, defined as the rate at which enactments complete. We ran the experiment on a single Linux computer, using tc-netem to simulate random packet loss. For each variation and each level of packet loss, we ran ten iterations of one minute each. In each iteration, MERCHANT generates a new
PO every 5 milliseconds (simulating external customers), with the POs having a uniformly distributed number of items between 1 and 4. Enactment timeouts were disabled, and the merchant's retry policy was configured to resend its messages every second until it received the corresponding Packed message.

Hypothesis Under random packet loss, UDP+Retry completes more enactments over a given duration than both MQTT variations and Plain UDP.

Figure 6 shows the results of our experiment as a graph of the average throughput (enactments completed per second) over packet loss probability. Each plot shows the mean of ten iterations, with error bars showing the sample standard deviation.

The results support our hypothesis. Plain UDP drops rapidly and consistently in throughput, since any message loss prevents an enactment from completing. In contrast, UDP+Retry has a consistently higher throughput under packet loss. Since Plain UDP has less overhead and both initiate enactments at the same rate, the difference is in their reliability: UDP+Retry recovers some enactments that would otherwise be lost.

Both MQTT variations suffer an initial drop in throughput at low packet loss, possibly due to TCP backoff. Their throughput changes very little up to $20 \%$ loss, demonstrating reliability superior to Plain UDP and the benefits of TCP's ability to batch multiple messages in one packet. However, both MQTT variations drop precipitously in throughput starting at $20 \%$ and fail to complete any enactments after $50 \%$ loss. At every packet loss rate, UDP+Retry has a higher throughput than either MQTT variation. Since TCP on Linux defaults to retrying packet transmissions for longer than the one-minute sample duration, and UDP+Retry never gives up, the 
difference in throughput is not due to lost enactments but efficiency. UDP+Retry is more efficient than MQTT's infrastructure-level retry solutions because it can detect losses without requiring message acknowledgments and no enactment blocks the progress of another.

\section{CONCLUSION}

PoT differs from existing work on protocol languages and deriving endpoint representations, e.g., [1, 3], in its use of an information-based representation for protocols. The PoT representation is uniquely compatible with asynchrony and naturally addresses correlation problems. PoT is also directly of practical value as it enables the generation of agent (endpoint) skeletons in NodeRED and, through them, the enforcement of protocol constraints.

PoT accommodates heterogeneity; an application may involve both PoT agents (implemented as described in Section VI) and non-PoT agents. PoT agents are guaranteed to be compliant with the protocol, but nonPoT agents can also be compliant. If a non-PoT agent is not compliant, however, the PoT agent's adapter provides some protection by recognizing and rejecting incoming messages that fail integrity.

PoT's basis in information protocols supports application-level retransmission of messages-a fault tolerance mechanism. PoT could be extended to support a wider and more sophisticated variety of such mechanisms. For example, retransmission could be adaptive: an agent could learn expected arrival times for messages from other agents relative to other messages and use that knowledge to determine when to resend a message. Future extensions should improve ease of use, flexibility, and robustness against various faults or malicious behavior until decentralized programming is no longer considered difficult but properly understood as the best way to improve fault tolerance and create scalable dynamic systems.

PoT opens up the possibility of expressing deeper expectations between parties, such as norms [2]. Just as PoT tackles expectations about the information exchanged, norms would capture the meaning of that information, such as whether one party committed to doing something for another or prohibited the other party from doing something. We could evaluate whether such a commitment or prohibition was satisfied or violated and whom to hold to account for it. Such models can enable greater social intelligence, paving the path to superior programming models for autonomy, flexibility, and reusability. 


\section{ACKNOWLEDGMENTS}

Thanks to the anonymous reviewers for helpful comments. Christie, Smirnova, and Chopra were supported by EPSRC grant EP/N027965/1 (Turtles). Christie and Singh were partially supported by the National Science Foundation under grant IIS-1908374.

\section{REFERENCES}

[1] M. Baldoni, C. Baroglio, and F. Capuzzimati, "A commitment-based infrastructure for programming socio-technical systems," ACM Transactions on Internet Technologies, vol. 14, no. 4, pp. 23:1-23:23, Dec. 2014.

[2] A. K. Chopra and M. P. Singh, "Custard: Computing norm states over information stores," in Proc. AAMAS. IFAAMAS, 2016, pp. 1096-1105.

[3] A. Ferrando, M. Winikoff, S. Cranefield, F. Dignum, and V. Mascardi, "On enactability of agent interaction protocols: Towards a unified approach," in Proc. EMAS, LNCS 12058, Springer, 2019, pp. 43-63.

[4] OASIS, "MQTT 3.1.1 specification document," Oct. 2014, OASIS Standard; http://docs.oasis-open. org/mqtt/mqtt/v3.1.1/os/mqtt-v3.1.1-os.pdf.

[5] C. Peltz, "Web service orchestration and choreography," IEEE Computer, vol. 36, no. 10, pp. 46-52, Oct. 2003.

[6] J. Postel, "User datagram protocol," $R F C$, vol. 768, pp. 1-3, 1980. [Online]. Available: https: //tools.ietf.org/html/rfc768

[7] J. H. Saltzer, D. P. Reed, and D. D. Clark, "Endto-end arguments in system design," ACM Transactions on Computer Systems, vol. 2, no. 4, pp. 277-288, Nov. 1984.

[8] Z. Shelby, K. Hartke, and C. Bormann, "The Constrained Application Protocol (CoAP)," IETF, RFC
7252, Jun. 2014, proposed standard; https://tools. ietf.org/html/rfc7252.

[9] S. Sicari, A. Rizzardi, and A. Coen-Porisini, "Smart transport and logistics: A Node-RED implementation," Internet Technology Letters, vol. 2, no. 2, p. e88, 2019.

[10] M. P. Singh, "Information-driven interactionoriented programming: BSPL, the Blindingly Simple Protocol Language," in Proc. AAMAS, IFAAMAS, 2011, pp. 491-498.

[11] M. P. Singh and A. K. Chopra, "The Internet of Things and multiagent systems: Decentralized intelligence in distributed computing," in Proc. ICDCS. IEEE, Jun. 2017, pp. 1738-1747, Blue Sky Thinking Track.

\section{AUTHOR Bios}

Samuel H. Christie $\mathbf{V}$ is a $\mathrm{PhD}$ student at NC State University and a Research Associate at the School of Computing and Communications at Lancaster University, UK. Contact him at schrist@ncsu.edu.

Daria Smirnova was a Research Associate at the School of Computing and Communications at Lancaster University, UK.

Amit K. Chopra is a Senior Lecturer in the School of Computing and Communications at Lancaster University, UK. Contact him at amit.chopra@lancaster.ac.uk.

Munindar P. Singh is a Professor in Computer Science and a co-director of the Science of Security Lablet at NC State University. Singh is an IEEE Fellow, a AAAI fellow, and a former Editor-in-Chief of IEEE Internet Computing and ACM Transactions on Internet Technology. Contact him at singh@ncsu.edu. 